\title{
CULTURA DE SEGURANÇA NUM SERVIÇO DE URGÊNCIA: PERCEÇÃO DOS PROFISSIONIAS DE SAÚDE- REVISÃO SISTEMÁTICA DA LITERATURA
}

\author{
Ana Martins Diz ${ }^{1}$ e Pedro Bernardes Lucas ${ }^{2}$ \\ ${ }^{1}$ Centro Hospitalar de Lisboa Ocidental, Portugal. ana_diz1@hotmail.com \\ ${ }^{2}$ Escola Superior de Enfermagem de Lisboa, Portugal. prlucas@esel.pt
}

\begin{abstract}
Resumo. Introdução: A segurança do doente em cuidados de saúde é uma prioridade global, que preocupa todos os implicados que dele dependem, imperando a necessidade de se conhecer em profundidade a realidade dos contextos particulares, como o serviço de urgência, para se garantir uma cultura de segurança. Objetivo: Analisar a evidência científica acerca da perceção dos enfermeiros sobre a cultura de segurança no serviço de urgência. Métodos: Revisão sistemática da literatura. Os estudos selecionados foram do período entre 2014 e 2019. Foram cinco os artigos que formaram o corpus amostral. Resultados: As perceções dos enfermeiros revelam que o ambiente de trabalho, o trabalho em equipa e as questões relacionadas com a liderança dos superiores hierárquicos são fatores fundamentais para se poder ter uma cultura de segurança que garanta a qualidade dos cuidados prestados. $O$ aspeto mais negativo é a falta de cooperação entre os elementos da equipa. O fator mais valorizado para a garantia de uma cultura de segurança do doente foi o trabalho em equipa, garantindo a melhoria da assistência ao cliente, redução de eventos adversos e a prestação de cuidados de qualidade. Conclusão: As evidências contribuem para melhorar a qualidade dos cuidados prestados.
\end{abstract}

Palavras-chave: Segurança do Paciente; Serviço Hospitalar de Emergência; Percepções; Enfermeiros. PATIENT SAFETY CULTURE IN EMERGENCY SERVICES: HEALTH PROFESSIONAL'S
PERCEPTIONS - SYSTEMATIC REVIEW

Abstract. Introduction: Patient safety in healthcare is a global priority, which concerns all those involved who depend on it, requiring the need to know in-depth the reality of particular contexts, such as the emergency service, to ensure a culture of safety. Objective: To analyze the scientific evidence about nurses' perception of the safety culture in the emergency department. Methods: Systematic literature review. The selected studies were from the period between 2014 and 2019 . Five articles formed the sample corpus. Results: Nurses' perceptions reveal that the work environment, teamwork, and issues related to the leadership of superiors are fundamental factors to be able to have a safety culture that guarantees the quality of care provided. The most negative aspect is the lack of cooperation between the team members. The most valued factor in ensuring a culture of patient safety was teamwork, ensuring the improvement of customer care, reduction of adverse events, and the provision of quality care. Conclusion: Evidence contributes to improving the quality of care provided.

Keywords: Patient safety; Emergency Service, Hospital; Perceptions; Nurses.

\section{INTRODUÇÃO}

A melhoria na segurança do doente é um dos problemas mais críticos enfrentados pelos serviços de saúde do século XXI. A segurança do doente é o resultado de esforços coletivos que procuram evitar erros médicos ou eventos adversos evitáveis e, portanto, salvaguardar os doentes (Milligan, 2007; McFadden, Henagan \& Gowen, 2009). A segurança do doente é 
difícil de obter nos cuidados de saúde prestados no serviço de urgência devido à presença de doentes com condições complexas de alta acuidade, um ambiente de trabalho difícil de controlar e trabalho em equipa multidisciplinar que envolve frequentes transferências com potencial para falhas na comunicação (Alshyyab, FitzGerald, Dingle et al., 2019).

Os profissionais de saúde do serviço de urgência trabalham em condições de alta pressão, com múltiplas interrupções e restrições de tempo. Assim, há uma possibilidade aumentada de erros e desfechos desfavoráveis dos doentes. Embora haja muitas abordagens para melhorar a segurança dos pacientes, estabelecer uma cultura de segurança é considerada uma estratégia central (Alshyyab, FitzGerald, Dingle et al., 2019). O serviço de urgência é um ambiente de trabalho desafiador devido à elevada afluência e à rotatividade de doentes, falta de controlo sobre a sobrecarga de trabalho dos enfermeiros e dificuldades de comunicação. Assim, torna-se num ambiente vulnerável a riscos para os doentes, pondo em causa a sua segurança (Källberg, Ehrenberg, Florin, Östergren \& Göransson, 2017). Glickman, Mehrotra, Shea et al. (2016) verificaram que 8,5\% dos problemas identificados pelo doente estavam relacionados com a sua segurança. Constataram que o serviço de urgência, com um incidente adverso de $3 \%$, foi o terceiro local mais comum de incidentes adversos em hospitais. Um estudo americano concluiu que $53 \%$ dos incidentes ocorridos no serviço de urgência eram evitáveis (Thomas, Studdert, Burstin et al., 2000). Outro estudo em 62 serviços de urgência nos EUA encontrou uma taxa global de 4,1 de incidentes adversos por 100 doentes, dos quais 37\% eram evitáveis (Camargo, Tsai, Sullivan et al., 2012).

Uma das componentes estruturantes e essenciais da qualidade na prestação de cuidados de saúde é a segurança do doente, existindo atualmente um conjunto de evidências e um vasto conhecimento factual sobre as implicações que a segurança dos doentes ou a sua falta tem sobre as organizações, os seus profissionais e, principalmente, sobre os doentes que a elas recorrem (Camargo, Tsai, Sullivan et al., 2012). A qualidade está intimamente relacionada com a segurança dos cuidados, como garantia do sistema de saúde, onde uma das componentes críticas da gestão dessa qualidade e dos cuidados de enfermagem é a segurança dos doentes (Thomas, Studdert, Burstin et al., 2000). Por esta razão, deve aumentar-se a responsabilização pela qualidade de todos os níveis do sistema e implementar o envolvimento dos profissionais e das lideranças, conforme consta da estratégia nacional para a qualidade na saúde 2015-2020 (Fernandes \& Queirós, 2011). A liderança é a base para aumentar a qualidade dos cuidados e diminuir os custos em saúde. A obtenção de qualidade em saúde serve o propósito de alcançar os melhores resultados 
nos cuidados prestados aos doentes, quer na melhoria do bem-estar, como na satisfação com os cuidados, dentro de um sistema bem organizado, mantendo os custos a um nível mínimo. Para atingir este objetivo é necessário o empenho total e completo envolvimento dos profissionais de saúde e das organizações de saúde (Fernandes \& Queirós, 2011).

Deste modo, é fundamental analisar a perceção dos enfermeiros sobre a cultura de segurança no serviço de urgência, o que se constituiu como objetivo deste trabalho, traduzindo-se na seguinte questão de investigação: Qual a perceção dos enfermeiros sobre a cultura de segurança num serviço de urgência?

\section{MÉTODOS}

Esta revisão teve como referência a metodologia proposta pelo Joanna Briggs Institute para revisões (JBI, 2015; Peters et al, 2015), tendo como objetivo "Analisar a evidência científica acerca da perceção dos enfermeiros sobre a cultura de segurança num serviço de urgência." Esta revisão sistemática responde à pergunta e baseia-se nos elementos PCC (População, Conceito e Contexto) tendo em conta os critérios de inclusão. A questão de revisão é: Qual a perceção dos enfermeiros sobre a cultura de segurança num serviço de urgência? Foram pesquisadas bases de dados para revisão de estudos relacionados com a perceção dos enfermeiros sobre a cultura de segurança no serviço de urgência, datados entre janeiro de 2014 a outubro de 2019: CINAHL Complete; MEDLINE Complete; Nursing \& Allied Health Collection: Comprehensive; Cochrane Central Register of Controlled Trials; Cochrane Database of Systematic Reviews; Cochrane Methodology Register; Library, Information Science \& Technology Abstracts; MedicLatina, via EBSCOhost - Research Databases; BVS - Biblioteca Virtual da Saúde, PubMed, RCAAP - Repositórios Científicos de Acesso Aberto de Portugal. A estratégia inicial de pesquisa usou termos de pesquisa mapeados para o Medical Subject Headings (MeSH). Foram utilizados os seguintes descritores: \#1 MeSH Emergency department; \#2 MeSH Nurse's perception; \#3 MeSH Patient Safety; \#4 MeSH Safety culture e \#4 [\#1 AND \# 2 AND \#3 OR \#4] (explode all trees). 


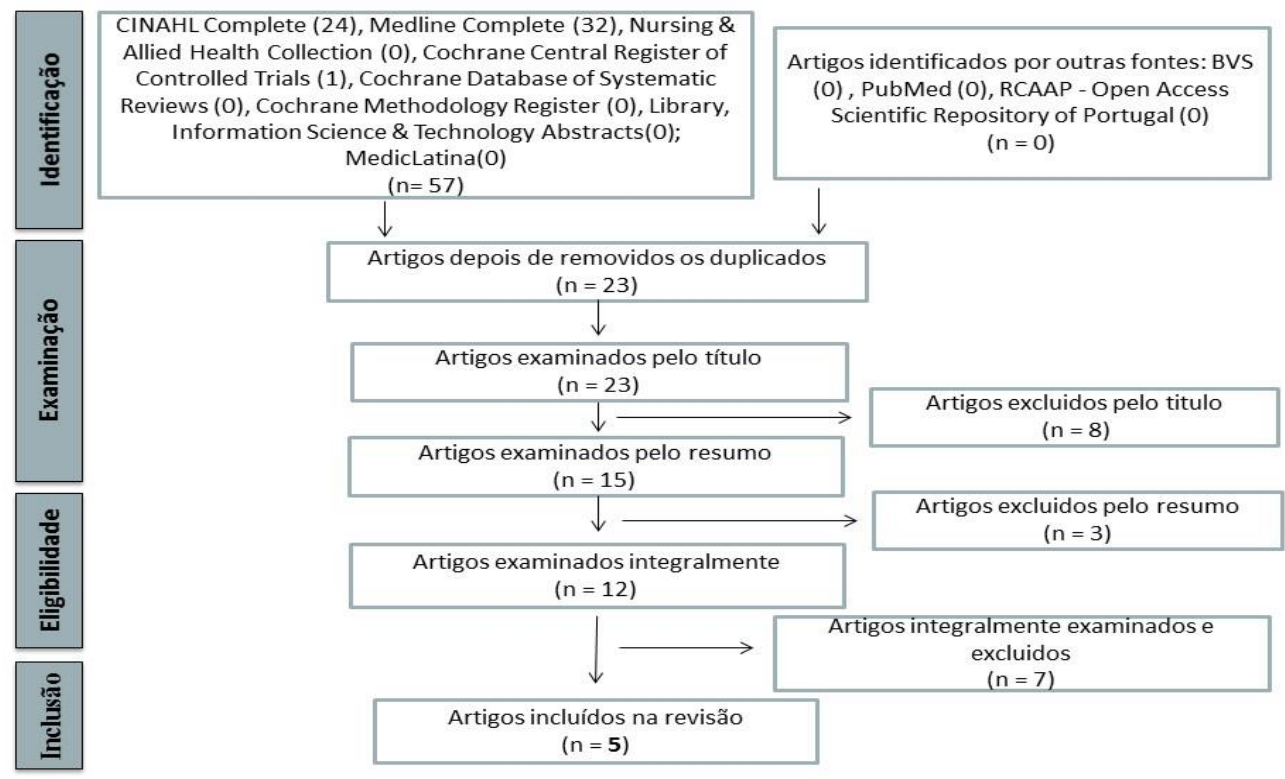

Figura 1. Diagrama com o processo de seleção dos estudos - PRISMA

Para a identificação de estudos relevantes dentro dos critérios de inclusão definidos foram realizadas pesquisas que compreenderam os estudos que datam de janeiro de 2014 a outubro de 2019, nos idiomas português, inglês e espanhol. Da sua conjugação com os operadores booleanos nos motores de busca para a obtenção dos textos completos das publicações que tinham sido identificadas, resultaram as seguintes formulações: (("Emergency department") AND " Nurse’s perception "]) AND "Patient Safety "]) OR " Safety culture"].

Definiram-se como critérios de inclusão: estudos publicados nas bases de dados anteriormente referidas, no espaço temporal de 2014-2019, apresentados em texto integral, nos idiomas inglês, português e espanhol, e cujo título e/ou resumo fizessem referência à perceção dos enfermeiros sobre a cultura de segurança no serviço de urgência.

O processo de triagem baseou-se no Reporting Items for Systematic Reviews and MetaAnalyses (PRISMA) para a realização de revisões sistemáticas (Moher, Liberati, Tetzlaff \& Altman, 2009) e na metodologia do Joanna Briggs Institute no que se refere às diretrizes de uma revisão sistemática (JBI, 2015; Peters et al, 2015), o que se assumiu como parte da estratégia de pesquisa. O texto completo de cada artigo foi revisto para determinar se satisfazia os critérios de inclusão do estudo por dois revisores com discussões entre ambos para obter consenso sobre a exclusão de artigos. 
Da pesquisa nas bases de dados, resultou a identificação de 57 artigos. Assim, numa primeira fase foi realizada uma leitura crítica e reflexiva dos títulos e dos resumos encontrados. Numa segunda fase, após a remoção dos artigos repetidos nas bases de dados ( $n=23$ ), estabeleceu-se uma amostra de 34 artigos. Depois da aplicação dos critérios de inclusão, ficou-se com uma amostra de 15 artigos para a elegibilidade. Posteriormente realizou-se a uma análise criteriosa dos artigos selecionados com exclusão de 5 artigos por não discriminarem entre os profissionais de saúde as perceções dos enfermeiros, tendo-se conservado 5 artigos dos quais se extraiu dos mesmos as evidências referentes à perceção dos enfermeiros sobre a cultura de segurança no serviço de urgência. Nesta etapa e de maneira a organizar-se toda a análise, fazendo-se uma leitura exploratória de cada artigo, identificaram-se, traduziram-se e transcreveram-se frases/palavras que correspondiam ao tema da perceção dos enfermeiros sobre a cultura de segurança no serviço de urgência. Com o intuito de sistematizar a informação dos artigos, os dados extraídos dos estudos foram compilados de forma descritiva numa tabela previamente elaborada, o que facilitou a identificação e reformulação das categorizações temáticas.

A Figura 1 especifica os resultados das etapas da análise, seguindo o modelo PRISMA Flow Diagram (Moher et al, 2009).

\subsection{Extração dos dados}

Os dados extraídos dos artigos foram alinhados com os objetivos e questões de pesquisa e respetivo instrumento indicados pela metodologia de análise desenvolvido pelo Joanna Briggs Institute (JBI, 2015; Peters et al, 2015). Ambos os revisores examinaram de forma independente os primeiros cinco estudos usando o formulário de dados, tendo depois reunido para determinarem se a abordagem aos dados extraídos é consistente com a pesquisa em questão (Levac, Colquhoun \& O’Brien, 2010).

Apresenta-se na tabela 1, o quadro síntese dos artigos incluídos na revisão. 
Tabela 1. Quadro síntese dos artigos incluídos na revisão

\begin{tabular}{|c|c|}
\hline $\begin{array}{l}\text { Título do artigo, autor, } \\
\text { ano e país }\end{array}$ & $\begin{array}{l}\text { Rigobello, M.C.G., Carvalho, R.E.F.L., Guerreiro, J.M., Motta, A.P.G., Atila, E., \& Gimenes, } \\
\text { F.R.E. (2017). The perception of the patient safety climate by professionals of the emergency } \\
\text { department }{ }^{(12)}\end{array}$ \\
\hline Tipo de estudo & Estudo quantitativo, descritivo, transversal. \\
\hline Objetivo do estudo & $\begin{array}{l}\text { Avaliar o clima de segurança do doente na perspectiva de profissionais de saúde que } \\
\text { trabalham no serviço de urgência de um hospital no Brasil. }\end{array}$ \\
\hline População e amostra & A amostra do estudo foi composta por 125 profissionais de saúde. \\
\hline Instrumentos utilizados & $\begin{array}{l}\text { The Safety Attitudes Questionnaire (SAQ) - Short Form 2006, validado e adaptado para o } \\
\text { português. }\end{array}$ \\
\hline Resultados & 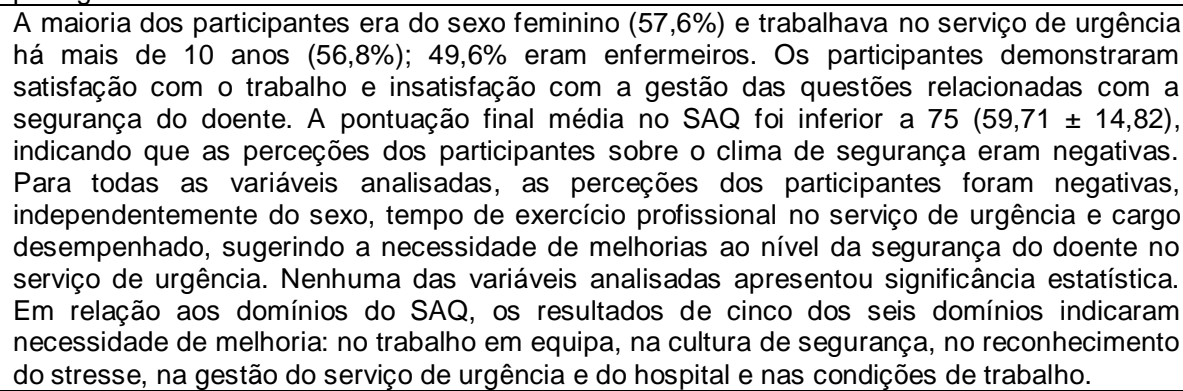 \\
\hline $\begin{array}{r}\text { Título do artigo, autor, } \\
\text { ano e país }\end{array}$ & $\begin{array}{l}\text { Burström, L., Letterstål, A., Engström, M-L., Berglund, A., \& Enlund, M. (2014). The patient } \\
\text { safety culture as perceived by staff at two different emergency departments before and after } \\
\text { introducing a flow-oriented working model with team triage and lean principles: a repeated } \\
\text { cross-sectional study }{ }^{(13)}\end{array}$ \\
\hline Tipo de estudo & Estudo transversal \\
\hline Objetivo do estudo & $\begin{array}{l}\text { Estudar a cultura de segurança do doente no serviço de urgência em dois hospitais diferentes } \\
\text { antes e depois de um projeto de melhoria da qualidade (QI) que visava aumentar a } \\
\text { segurança do doente. }\end{array}$ \\
\hline População e amostra & $\begin{array}{l}\text { Participaram vários grupos profissionais, sendo a maioria enfermeiros, com prevalência da } \\
\text { faixa etária dos } 25 \text { a } 44 \text { anos. Em todos os grupos, a maioria dos profissionais de saúde } \\
\text { possuía de } 1 \text { a } 5 \text { anos de experiência profissional, exceto os enfermeiros do hospital do } \\
\text { municipal que na maioria possuíam mais de } 21 \text { anos de experiência profissional. A duração } \\
\text { predominante dos anos de experiência profissional no serviço de urgência foi de } 1 \text { a } 5 \text { anos. }\end{array}$ \\
\hline Instrumentos utilizados & $\begin{array}{l}\text { Foi utilizado o questionário Hospital Survey on Patient Safety Culture (HSOPSC), antes e } \\
\text { depois de um projeto de melhoria da qualidade que visava melhorar a segurança do doente } \\
\text { no serviço de urgência de dois hospitais, um hospital municipal e um hospital universitário, em } \\
\text { duas cidades diferentes no centro da Suécia. }\end{array}$ \\
\hline Resultados & $\begin{array}{l}\text { No hospital municipal, foi observada uma diferença em duas dimensões, trabalho em equipa } \\
\text { e abertura de comunicação, com uma pontuação mais alta no acompanhamento do doente. } \\
\text { No hospital universitário, obteve-se um score mais alto no acompanhamento do doente. O } \\
\text { fator mais valorizado para a garantia de uma cultura de segurança do doente, em ambos os } \\
\text { hospitais, foi o trabalho em equipa. }\end{array}$ \\
\hline $\begin{array}{r}\text { Título do artigo, autor, } \\
\text { ano e país }\end{array}$ & $\begin{array}{l}\text { Alzahrani, N., Jones, R., Abdel-Latif, M.E. (2018). Attitudes of doctors and nurses toward } \\
\text { patient safety within emergency departments of two Saudi Arabian hospitals }{ }^{(14)}\end{array}$ \\
\hline Tipo de estudo & Estudo transversal \\
\hline Objetivo do estudo & $\begin{array}{l}\text { Investigar as atitudes de médicos e de enfermeiros em relação à cultura de segurança do } \\
\text { doente em serviços de urgência de dois hospitais sauditas. }\end{array}$ \\
\hline População e amostra & $\begin{array}{l}\text { Amostra constituída por } 503 \text { médicos e enfermeiros a exercerem funções no serviço de } \\
\text { urgência. }\end{array}$ \\
\hline Instrumen & Foi utilizado, para recolha de dados, o Safety Attitudes Questionnaire (SAQ) \\
\hline Resultados & $\begin{array}{l}\text { A pontuação média de cada dimensão do } S A Q \text { foi }<75 \% \text {, indicando que enfermeiros e } \\
\text { médicos geralmente tiveram menos atitudes positivas ao nível da segurança do doente, } \\
\text { particularmente nas dimensões de reconhecimento do stresse }(58,1 \%) \text { e perceções ao nível } \\
\text { da gestão hospitalar }(56,9 \%) \text {. Os enfermeiros demonstraram menor pontuação na perceção } \\
\text { em relação ao trabalho em equipa ( } p<0,01) \text {, enquanto os médicos pontuam menos nas } \\
\text { condiços de trabalho do que os enfermeiros }(p<0,01) \text {. Houve uma correlação significativa } \\
\text { entre o número de erros relatados e o clima de trabalho em equipa, satisfação com o trabalho } \\
\text { e condições de trabalho. }\end{array}$ \\
\hline $\begin{array}{r}\text { Título do artigo, } \\
\text { ano } e\end{array}$ & $\begin{array}{l}\text { Van Noord, I. V., Wagner, C., Van Dyck, C., Twisk, J. W. R., \& De Bruijne, M. C. (2014). Is } \\
\text { culture associated with patient safety in the emergency department? A study of staff } \\
\text { perspectives. (15). }\end{array}$ \\
\hline Tipo de estudo & Estudo transversal \\
\hline Objet & $\begin{array}{l}\text { Averiguar as associações entre as dimensões da cultura de segurança do doente e os níveis } \\
\text { de segurança do doente, conforme relatado pela equipa de um serviço de urgência, e } \\
\text { comparar essas associações entre enfermeiros e médicos. }\end{array}$ \\
\hline
\end{tabular}




\begin{tabular}{|c|c|}
\hline População e amostra & $\begin{array}{l}\text { Amostra constituída por } 480 \text { enfermeiros, } 159 \text { médicos e } 91 \text { outros profissionais de serviços } \\
\text { de urgência de } 33 \text { hospital centrais holandeses. }\end{array}$ \\
\hline $\begin{array}{r}\text { Instrumentos } \\
\text { utilizados }\end{array}$ & $\begin{array}{l}\text { Foi utilizado o questionário Hospital Survey on Patient Safety Culture (HSOPSC) para recolha } \\
\text { de dados. }\end{array}$ \\
\hline Resultados & $\begin{array}{l}\text { A maioria dos participantes trabalhava no serviço de urgência há menos de } 10 \text { anos. Em } \\
\text { análises não ajustadas, todas as dimensões da cultura de segurança do doente foram } \\
\text { associadas positivamente ao nível relatado de segurança do doente e seis dessas } \\
\text { associações com a segurança do doente foram estatisticamente significativas após o ajuste } \\
\text { (trabalho em equipa, frequência da notificação de eventos adversos, abertura de } \\
\text { comunicação, feedback sobre e aprender com os erros, suporte de gestão do hospital para a } \\
\text { segurança do doente). Foram encontradas diferenças entre as perceções dos enfermeiros e } \\
\text { médicos em duas dimensões (frequência de notificação de eventos adversos e suporte da } \\
\text { gestão do hospital em relação à segurança do doente). A perceção dos médicos em relação à } \\
\text { cultura de segurança do doente no serviço de urgência foi mais positiva do que a dos } \\
\text { enfermeiros, que consideram como aspeto mais negativo a falta de cooperação entre os } \\
\text { elementos da equipa, com interferência na segurança do doente. }\end{array}$ \\
\hline $\begin{array}{l}\text { Título do artigo, } \\
\text { autor, ano e país }\end{array}$ & $\begin{array}{l}\text { Tourani, S., Hassani, M., Ayoubian, A., Habibi, M., \& Zaboli, R. (2015). Analyzing and } \\
\text { prioritizing the dimensions of patient safety culture in emergency wards using the TOPSIS } \\
\text { technique }{ }^{(16)}\end{array}$ \\
\hline Tipo de estudo & Analítico-descritivo e transversal \\
\hline Objetivo do estudo & $\begin{array}{l}\text { Analisar os aspetos da cultura de segurança do doente no serviço de urgência de hospitais } \\
\text { afiliados à Universidade de Ciência Médica de Teerão. }\end{array}$ \\
\hline População e amostra & Amostra constituída por 270 médicos e enfermeiros \\
\hline $\begin{array}{r}\text { Instrumentos } \\
\text { utilizados }\end{array}$ & $\begin{array}{l}\text { Para a recolha de dados, foi utilizado o questionário padrão Hospital Survey on Patient Safety } \\
\text { Culture (HSOPSC) }\end{array}$ \\
\hline Resultados & $\begin{array}{l}\text { Houve uma relação significativa entre as dimensões desempenho, trabalho em equipa, } \\
\text { feedback, saber lidar com os erros e apoio das chefias }(p \leq 0,05) \text {. O score total da cultura de } \\
\text { segurança do doente na maioria dos hospitais situava-se num nível médio de } 3 \text {. A pontuação } \\
\text { máxima foi } 5 \text {. Os resultados da análise multivariada da tomada de decisão indicaram que os } \\
\text { fatores humanos, de gestão das chefias, organizacionais e ambientais estavam no topo das } \\
\text { prioridades em ordem decrescente. Os enfermeiros, na sua maioria, indicaram que os fatores } \\
\text { humanos são os mais eficazes e importantes na melhoria da cultura de segurança do doente } \\
\text { no serviço de urgência. Os resultados mostraram que as expectativas e o desempenho do } \\
\text { superior hierárquico e o apoio da gestão hospitalar estavam num nível médio; } 30 \% \text { dos } \\
\text { participantes relataram que o seu superior hierárquico não presta atenção às suas } \\
\text { recomendações para melhorar a segurança dos doentes e } 52 \% \text { dos enfermeiros } \\
\text { argumentaram que o seu superior hierárquico os apoia quando eles executam uma tarefa } \\
\text { baseada nos princípios da cultura de segurança do doente; } 17 \% \text { referiram a melhoria da } \\
\text { segurança dos doentes se assume como um fator crítico que tem a ver com todos os níveis } \\
\text { de gestão do serviço de urgência. Ou seja, cada superior hierárquico deve criar um ambiente } \\
\text { de suporte na sua secção, como a implementação de programas de segurança e cursos de } \\
\text { formação. }\end{array}$ \\
\hline
\end{tabular}

\section{DISCUSSÃO DOS RESULTADOS}

Os artigos analisados são unânimes ao considerarem que o conhecimento das perceções dos profissionais de saúde, mormente dos enfermeiros, sobre a cultura de segurança do doente no contexto de serviço de urgência contribui para a melhoria da assistência ao cliente, reduz os eventos adversos e pode concentrar esforços para uma prestação de cuidados de qualidade. Promover a segurança do doente e do trabalho são dois principais desafios para as organizações hospitalares. Ao tentar melhorar esses dois resultados de forma sinérgica, devem ser consideradas as condições psicossociais de trabalho, a liderança e as perceções da cultura de segurança do doente. Os estudos mostraram que esses tópicos-chave estão inter-relacionados e formam uma base crítica para promover a segurança do doente no serviço de urgência. 
Para se gerir efetivamente esses desafios, recomenda-se às organizações de saúde o desenvolvimento de uma cultura de segurança (Van Noord, Wagner, Van Dyck, Twisk \& De Bruijne, 2014; Tourani, Hassani, Ayoubian, Habibi \& Zaboli, 2015). Uma cultura de segurança das organizações de saúde refere-se ao produto de valores, atitudes, perceções, competências e padrões de comportamento de indivíduos e grupos que determinam o compromisso, o estilo e a proficiência da gestão de segurança e da saúde de uma organização (Wagner et al., 2019). Os serviços de urgência com uma cultura de segurança do doente positiva caracterizam-se por comunicações baseadas na confiança mútua, em perceções compartilhadas da importância da segurança e na confiança na eficácia de medidas preventivas (Burström, Letterstål, Engström, Berglund \& Enlund, 2014).

A cultura de segurança do doente reflete como a segurança é vista e tratada, no caso concreto no serviço de urgência. No entanto, nem sempre as perceções dos enfermeiros são positivas (Rigobello, et al, 2017), independentemente do sexo, tempo de exercício profissional no serviço de urgência e cargo desempenhado, sugerindo a necessidade de melhorias a nível da segurança do doente no serviço de urgência, a nível do trabalho em equipa, na cultura de segurança, no reconhecimento do stresse, na gestão do serviço de urgência e do hospital e nas condições de trabalho. É transversal a todos os estudos analisados a valorização do trabalho em equipa e a abertura de comunicação, como garantia de uma cultura de segurança do doente (Van Noord et al., 2014; Tourani et al., 2015; Rigobello, Carvalho, Guerreiro, Motta, Atila \& Gimenes, 2017; Alzahrani, Jones \& Abdel-Latif, 2018).

No estudo de Alzahrani et al. (2018), as atitudes de segurança dos médicos e enfermeiros de serviços de urgência de hospitais sauditas são menos positivas e estão correlacionadas com o número de erros relatados. As intervenções de treino em segurança e o suporte da gestão parecem ser os caminhos mais prováveis para melhorar as atitudes e o desempenho dos profissionais de saúde em relação à segurança do doente. No estudo de Alzahrani et al. (2018) houve uma correlação significativa entre o número de erros relatados e o clima de trabalho em equipa, satisfação com o trabalho e condições de trabalho, sendo estes fatores valorizados pelos enfermeiros como fundamentais para uma verdadeira cultura de segurança do doente no serviço de urgência. No estudo de Tourani et al. (2015) houve uma relação significativa entre as dimensões desempenho, trabalho em equipa, feedback, saber lidar com os erros e apoio das chefias. O score total da cultura de segurança do doente na maioria dos serviços de urgência dos hospitais estudados situava-se num nível médio de 3. 
Os enfermeiros, na sua maioria, indicaram que os fatores humanos são os mais eficazes e importantes na melhoria da cultura de segurança do doente no serviço de urgência. Os resultados mostraram que as expectativas e o desempenho do superior hierárquico e 0 apoio da gestão hospitalar estavam num nível médio; $30 \%$ dos participantes relataram que o seu superior hierárquico não presta atenção às suas recomendações para melhorar a segurança dos doentes e $52 \%$ dos enfermeiros argumentaram que o seu superior hierárquico os apoia quando eles executam uma tarefa baseada nos princípios da cultura de segurança do doente; $17 \%$ referiram a melhoria da segurança dos doentes se assume como um fator crítico que tem a ver com todos os níveis de gestão do serviço de urgência. Ou seja, cada superior hierárquico deve criar um ambiente de suporte na sua secção, como a implementação de programas de segurança e cursos de formação. Os fatores humanos foram os mais eficazes e importantes na melhoria da segurança do doente no serviço de urgência, sugerindo a necessidade de se prestar mais atenção a esses fatores na implementação da melhoria da cultura de segurança do doente. Treino, boas condições dos ambientes organizacionais e fatores ambientais motivadores foram as principais medidas que devem ser tidas em consideração pelos enfermeiros. Estudos anteriores mostraram que as perceções dos enfermeiros sobre as condições psicossociais do trabalho e a cultura de segurança variam, embora funcionem no mesmo ambiente (Kristensen et al., 2015; Aiken, Cimiotti, Sloane, Smith, Flynn \& Neff, 2011). Outros estudos também identificaram estreitas relações entre o ambiente de trabalho e a cultura de segurança (McHugh et al., 2016; Aiken et al., 2014). Assim, pode assumir-se que a melhoria das condições de trabalho dos profissionais de saúde também leva a uma melhor cultura de segurança. Para se otimizar a cultura de segurança do doente é fundamental também garantir a segurança do ambiente, as condições psicossociais do trabalho, a liderança dos superiores hierárquicos.

\section{CONCLUSÕES}

O conhecimento das perceções dos enfermeiros sobre a cultura de segurança do doente no serviço de urgência contribui para a melhoria dos cuidados de saúde, reduzindo os eventos adversos e pode concentrar esforços para melhorar a qualidade do atendimento prestado aos clientes. Um dos direitos humanos mais claros é o direito de estar a salvo dos possíveis riscos no decorrer da prestação de cuidados de saúde. A segurança do doente tem vindo a ganhar destaque em todo o mundo e, todos os dias, os enfermeiros no serviço de urgência enfrentam vários desafios em como oferecer uma assistência segura aos clientes. Conhecer a perceção dos enfermeiros sobre a segurança do doente é uma das principais ferramentas 
para se garantir uma cultura de segurança no serviço de urgência. As evidências revelam que ainda persistem perceções negativas dos enfermeiros a exercerem em serviços de urgência, considerando que o ambiente de trabalho, o trabalho em equipa e as questões relacionadas com a liderança dos superiores hierárquicos são fatores fundamentais para se poder ter uma cultura de segurança que garanta a qualidade dos cuidados prestados. Espera-se que esta revisão sistemática contribua para evidenciar o estado atual do conhecimento acerca do tema e que os resultados encontrados se assumam como principais linhas orientadoras para o desenvolvimento e aplicabilidade de formação para os enfermeiros na área da segurança do doente.

\section{REFERÊNCIAS}

Aiken, L. H., Cimiotti, J. P., Sloane, D. M., Smith, H. L., Flynn, L., \& Neff, D. F. (2011). Effects of nurse staffing and nurse education on patient deaths in hospitals with different nurse work environments. Medical Care; 49, 1047-53. https://doi.org/10.1097/MLR.0b013e3182330b6e

Aiken, L.H., Sloane, D.M., Bruyneel, L., Heede, K., Griffiths, P., Busse, R. et al. (2014). Nurse staffing and education and hospital mortality in nine European countries. A retrospective observational study. Lancet, 383, 1824-30. https://doi.org/10.1016/S0140-6736(13)62631-8

Alshyyab, M. A., FitzGerald, G., Dingle, K., Ting, J., Bowman, P., Kinnear, F. et al (2019). Developing a conceptual framework for patient safety culture in emergency department: A review of the literature. The International Journal of Health Planning and Management; 34, 42-55. https://doi.org/10.1002/hpm.2640

Alzahrani, N., Jones, R. \& Abdel-Latif, M. E. (2018). Attitudes of doctors and nurses toward patient safety within emergency departments of two Saudi Arabian hospitals. BMC Health Services Research; 18, 736, 2-7. https://doi.org/10.1186/s12913-018-3542-7

Burström, L., Letterstål, A., Engström, M-L., Berglund, A., \& Enlund, M. (2014). The patient safety culture as perceived by staff at two different emergency departments before and after introducing a flow-oriented working model with team triage and lean principles: a repeated cross-sectional study. BMC Health Services Research, 14, 296, 2-12. https://doi.org/10.1186/1472-6963-14-296

Camargo, C. A., Tsai, C. L., Sullivan, A. F. Cleary, P. D., Gordon, J. A., Guadagnoliet, E. et al. (2012). Safety climate and medical errors in 62 US emergency departments. Annals of Emergency Medicine; 60(5):e555-e520. http://dx.doi.org/10.1016/j.annemergmed.2012.02.018

Fernandes, A. M. M. L., \& Queirós, P. J. P. (2011). Cultura de Segurança do Doente percecionada por enfermeiros em hospitais distritais portugueses. Revista de Enfermagem Referência, (4), 37-48. http://www.scielo.mec.pt/pdf/ref/vserllln4/serllIn4a04.pdf

Glickman, S. W., Mehrotra, A., Shea, C. M. Strickler, M. C., Pabers, S., Larson, J., et al. (2016). A patient reported approach to identify medical errors and improve patient safety in the emergency department. Journal of Patient Safety; 20(11) 2. DOI: 10.1097/pts.0000000000000287

Joanna Briggs Institute (2015). Joanna Briggs Institute Reviewers' Manual 2015: Methodology for JBI Scoping Reviews. Adelaide, Australia: The Joanna Briggs Institute. http://joannabriggs.org/assets/docs/sumari/Reviewers-Manual_Methodology-for-JBI-ScopingReviews_2015_v2.pdf 
Källberg, A. S., Ehrenberg, A., Florin, J., Östergren, J., \& Göransson, K. E. (2017). Physicians' and nurses' perceptions of patient safety risks in the emergency department. International Emergency Nursing; 33, 14-19. https://doi.org/10.1016/j.ienj.2017.01.002

Kristensen, S., Hammer, A., Bartels, P., Suñol, R., Groene, O., Thompson, C. A. et al. (2015). Quality management and perceptions of teamwork and safety climate in European hospitals. International Journal for Quality in Health Care; 27, 499-506. https://doi.org/10.1093/intqhc/mzv079

Levac, D., Colquhoun, H. \& O’Brien, K. K. (2010). Scoping studies: advancing the methodology. Implement Science; 5(69): 1-9. https://doi.org/10.1186/1748-5908-5-69

McFadden, K. L., Henagan, S. C., \& Gowen, C. R. (2009). The patient safety chain: transformational leadership's effect on patient safety culture, initiatives, and outcomes. Journal of Operations Management; 27(5), 390-404. https://doi.org/10.1016/j.jom.2009.01.001

McHugh, M. D., Rochman, M. F., Sloane, D. M., Berg, R. A., Mancini, M. E., Nadkarni, V. M. et al. (2016). Better nurse staffing and nurse work environments associated with increased survival of in-hospital cardiac arrest patients. Medical Care. 2016;54: 74-80. https://doi.org/10.1097/MLR.0000000000000456

Milligan, F. J. (2007). Establishing a culture for patient safety-the role of education. Nurse Education Today; 27(2), 95-102.

Moher, D., Liberati, A., Tetzlaff, J., \& Altman, D. G. (2009). Preferred reporting items for systematic reviews and meta-analyses: the PRISMA statement. Annals of Internal Medicine; 151(4), 264-269. DOI: 10.7326/0003-4819-151-4-200908180-00135

Peters, M., Godfrey, C., Mclnerney, P., Soares, C. B., Khalil, H. \& Parker, D. (2015). Methodology for JBI reviews. The Joanna Briggs Institute Reviewers' Manual. Adelaide, Australia: The Joanna Briggs Institute; 1-24.

Rigobello, M. C. G., Carvalho, R. E. F. L., Guerreiro, J. M., Motta, A. P. G., Atila, E., \& Gimenes, F. R. E. (2017). The perception of the patient safety climate by professionals of the emergency department. International Emergency Nursing, 33, 1-6. http://dx.doi.org/10.1016/j.ienj.2017.03.003

Thomas, E. J., Studdert, D. M., Burstin, H. R., Orav, E. J., Zeena, T., Williams, E. J., Howard, K. M., Weiler, P. C. \& Brennan, T. (2000). Incidence and types of adverse events and negligent care in Utah and Colorado. Medical Care.; 38(3), 261-271.

Tourani, S., Hassani, M., Ayoubian, A., Habibi, M., \& Zaboli, R. (2015). Analyzing and prioritizing the dimensions of patient safety culture in emergency wards using the TOPSIS technique. Global Journal of Health Science; 7(4):143-150. http://dx.doi.org/10.5539/gjhs.v7n4p143

Van Noord, I. V., Wagner, C., Van Dyck, C., Twisk, J. W. R., \& De Bruijne, M. C. (2014). Is culture associated with patient safety in the emergency department? A study of staff perspectives. International Journal for Quality in Health Care; 26(1), 64-70. https://doi.org/10.1093/intqhc/mzt087

Wagner, A., Rieger, M. A., Manser, T., Sturm, H., Hardt, J., Martus, P., Lessing, C., Hammer, A. \& WorkSafeMed Consortium (2019). Healthcare professionals' perspectives on working conditions, leadership, and safety climate: a cross-sectional study. BMC Health Services Research; 19, 53. https://doi.org/10.1186/s12913-018-3862-7 